\title{
Stochastic structure of annual discharges of large European rivers
}

\author{
Milan Stojković ${ }^{1 *}$, Stevan Prohaska ${ }^{1}$, Jasna Plavšić ${ }^{2}$ \\ ${ }^{1}$ Jaroslav Černi Institute for the Development of Water Resources, Jaroslava Černog 80, Belgrade, Serbia. \\ ${ }^{2}$ University of Belgrade, Faculty of Civil Engineering, Bulevar Kralja Aleksandra 63, Belgrade, Serbia. \\ ${ }^{*}$ Corresponding author. E-mail: milan.stojkovic@jcerni.co.rs
}

\begin{abstract}
Water resource has become a guarantee for sustainable development on both local and global scales. Exploiting water resources involves development of hydrological models for water management planning. In this paper we present a new stochastic model for generation of mean annul flows. The model is based on historical characteristics of time series of annual flows and consists of the trend component, long-term periodic component and stochastic component. The rest of specified components are model errors which are represented as a random time series. The random time series is generated by the single bootstrap model (SBM). Stochastic ensemble of error terms at the single hydrological station is formed using the SBM method. The ultimate stochastic model gives solutions of annual flows and presents a useful tool for integrated river basin planning and water management studies. The model is applied for ten large European rivers with long observed period. Validation of model results suggests that the stochastic flows simulated by the model can be used for hydrological simulations in river basins.
\end{abstract}

Keywords: Stochastic model; Flow simulation; Long-term periodicity; Single bootstrap model SBM.

\section{INTRODUCTION}

Systematic streamflow gauging in the modern era began rather late. The longest time series are available in Europe but they do not exceed 200 years. Long-term fluctuations of hydrological and meteorological quantities have been the object of attention of hydrologists and climatologists since the beginning of the 20th century.

Long-term river flow variations are generally studied by trend analysis, where the Mann-Kendall trend test is often used to identify the trend (Machiwal and Jha, 2006; Salas et al., 1980). The trend analysis may be misleading because long-term flow fluctuations are disregarded. As such, it is necessary to use several full time series cycles to assess the trend. There may be an additional problem if spatial correlation is disregarded. It might lead to reduction in the trend assessment data (Douglas et al., 2000). Khaliq et al. (2009) presented ways to remove serial correlation from hydrologic time series, such as pre-whitening, variance correction and block bootstrap. Apart from trend analysis, long-term variation can be studied by moving statistical distribution (Hanggi and Weingartner, 2011) and multitemporal analysis (Hannaford et al., 2013).

On the global scale, upward annual river flow trends have been registered in parts of the northern hemisphere, such as North America and northern Europe (Kundzewicz et al., 2008). Downward trends have been reported in parts of western and southern Africa, southern Europe and the Middle East (Milly et al., 2005). Birsan et al. (2005) performed trend analysis in 48 Swiss watersheds. They identified the significant upward annual flow trends in the winter and spring, and registered downward trends during the summer. Stahl et al. (2010) revealed downward annual flow trends in southern and eastern Europe, and upward trends in other parts of Europe.

Hurst (1951) examined the special behavior of hydrological time series in a study of long-term water availability. The phenomenon is based on the tendency of a dry year to group within a long dry period, as well as a wet year to group within a wet period. Box and Jenkins (1970) proposed methods for modeling long-term flow variations based on linear autoregression models while Yevjevich (1972) studied flow variability using the period- gram (discrete spectrum). Further developments in spectrum estimation involved the multitaper spectrum (Mudelsee, 2010) and the wavelet transform method (Labat et al., 2004).

The long-term flow fluctuation in European rivers was revealed by Probst and Tardy (1987), Pekarova et al. (2006) Pekarova and Pekar (2006), Labat (2006) and Fendekova et al. (2014). They discussed shifting of multiple-year dry and wet in the river basin.

The present research addresses mean annual flows of ten large European rivers. The objective is to establish the stochastic structure and model the annual flow series $Q_{m}$, assuming that the time series is non-stationary and composed of the sum of the following components: trend component $Q_{T}$, periodic component $Q_{p}$, stochastic component $Q_{A R}$ and the error term $\varepsilon$. In order to model the periodic component, Fourier's transformation of the time series is applied. The stochastic component is modeled by the linear autoregressive models. The error term is generated by the single bootstrap model (SBM) with resampling of historical data. The aim is to create a stochastic ensemble of simulations which could be used in water planning and management.

\section{Data sets}

Institute for the Development of Water Resources "Jaroslav Černi" maintains a database of hydrological and meteorological parameters of the longest time series on record worldwide. It was compiled using data provided by the Institute of Hydrology SAS from Bratislava, Slovakia, and the Global Runoff Data Centre attached to the German Federal Institute of Hydrology from Koblenz, Germany. The longest time series of mean annual flows were selected to assess runoff associated with large European rivers. The hydrological stations used in the study are listed in Table 1.

\section{METHODOLOGY}

In the analysis of the structure of the time series, the trend of time series is an important feature. Many trend analyses are based on the well-known Mann-Kendall trend test (Machiwal 
Table 1. Hydrological stations at the European rivers used in the study with basic information on the stations and the long-term mean annual flow $Q$.

\begin{tabular}{|c|c|c|c|c|c|}
\hline River and Station: & Country: & Basin area $\left(\mathrm{km}^{2}\right)$ : & Total years: & First year: & $Q\left(\mathrm{~m}^{3} / \mathrm{s}\right)$ \\
\hline Elbe - Decin & $\mathrm{CZ}$ & 51123 & 157 & 1851 & 305.3 \\
\hline Danube - Bratislava & SLO & 131331 & 102 & 1900 & 2050 \\
\hline Rhine - Cologne & $\mathrm{D}$ & 144232 & 193 & 1816 & 2088 \\
\hline Weser - Vlotho & $\mathrm{D}$ & 17618 & 189 & 1820 & 168.0 \\
\hline Inn - Wasserburg & $\mathrm{D}$ & 11983 & 182 & 1826 & 350.5 \\
\hline Salzach - Burghausen & $\mathrm{D}$ & 6649 & 182 & 1826 & 258.6 \\
\hline Danube - Orsova & RO & 576232 & 151 & 1840 & 5602 \\
\hline Vuoksi - Tainionkoski & SF & 61061 & 158 & 1847 & 592.1 \\
\hline Northern Dvina - Usti-Pinega & RUS & 348000 & 123 & 1881 & 3331 \\
\hline Nemunas - Smalininkai & LT & 81200 & 192 & 1812 & 536.9 \\
\hline
\end{tabular}

and Jha, 2006), a nonparametric test, based on the data ranks within the time series. This approach is used in this study to determine the significance of the annual flow trends, either linear or nonlinear. In order to model the trend and extract it from the rest of the time series, the trend in the time series is assumed to be linear. Significance of the parametric linear trend slope is tested for all annual flow series using the parametric Kendall-Stuart test (Kendall and Stuart, 1966). The hypothesis $H_{0}$ that there is no significant linear trend is rejected at significance level of $\alpha=5 \%$ if the identified trend slope $\beta$ is greater than:

$|\beta|>1.96 \sqrt{\frac{12}{N^{3}}}$

where $N$ is length of the annual flow series $Q$.

For the long-term periodicity analysis, the flow time series have to be smoothed in order to facilitate recognition and extraction of the periodic component with oscillation period that govern alternating multiple-year dry and wet periods in the river basin. For this purpose the LOESS smoothing technique is applied (Stojković et al., 2012). If the time series exhibits the long-term periodicity, it would be identified by the Hurst analysis. In this paper the well-known $R / S$ analysis is applied (Blasco and Santamaria, 1996).

The inherent assumption of spectral analysis is that the considered time series can be broken up into a set of finite or infinite sums of trigonometric sine and cosine functions (Shuster, 1887). The periodic component $Q_{P}$ can then be expressed as:

$Q_{P}(t)=\sum_{i=1}^{q}\left(a_{i} \sin \left(2 \pi f_{i} t\right)+b_{i} \cos \left(2 \pi f_{i} t\right)\right) \quad t=1, \ldots, N$,

where $t$ is the time step, $f_{i}$ is the frequency, $a_{i}$ and $b_{i}$ are the Fourier coefficients (amplitudes) and $q$ is the number of significant harmonics. The Fourier analysis provides the coefficients $a_{i}$ and $b_{i}$ as:

$a_{i}=\frac{2}{N} \sum_{t=1}^{N} Z_{i} \cos \left(2 \pi f_{i} t\right), b_{i}=\frac{2}{N} \sum_{t=1}^{N} Z_{i} \sin \left(2 \pi f_{i} t\right)$.

The periodogram is comprised of $q=(N-1) / 2$ discrete values if $N$ is an odd number and $q=N / 2$ if $N$ is an even number. The intensity of periodicity is determined as:
$I\left(f_{i}\right)=\frac{N}{2}\left(a_{i}^{2}+b_{i}^{2}\right) ; i=1,2, \ldots, q$

The intensity of periodicity is computed using Eq. (4) for discrete frequencies $f_{i}=i / N$ (Yevjevich, 1972), where $f_{i}$ is the frequency of the $i$-th period and $i$ is the serial number of the period $(i=1,2, \ldots, q)$. The Fourier analysis was used to determine the coefficients $a_{i}$ and $b_{i}$ (Eq. 3). In the next step, the Fisher's test and the relative cumulative periodogram $(R C P)$ (Pekarova et al., 2003; Yevjevich, 1972) are applied to define significant periods.

The periodicity of annual flows characterized by short-term periods is analyzed by applying the autoregressive models (Box and Jenkins, 1970). This component of annual flows is referred to as stochastic component. When the linear trend $Q_{T}$ and the long-term periodic component $Q_{P}$ are removed from the annual flow time series $Q$, the remaining part presents the stochastic and the random component. The remainder of the time series is called the residual flow $Q_{R}$, derived as follows:

$Q_{R}(t)=Q(t)-Q_{T}(t)-Q_{P}(t)$

The residual flow series $Q_{R}$ is considered as a stochastic stationary process $x_{t}$, which represents the standardized series $x_{t}=Q_{R} / \sigma_{R}$ where $\sigma_{R}$ is standard deviation of $Q_{R}$. The $\operatorname{AR}(p)$ models were used to model stochastic component from the standardized residuals $x_{t}$ (Box and Jenkins, 1970):

$x_{t}(t)=\phi_{1} \cdot x_{t}(t-1)+\phi_{2} \cdot x_{t}(t-2)+\ldots+\phi_{p} \cdot x_{t}(t-p)$.

The above equation shows that the $\operatorname{AR}(p)$ model represents the weighted sum of the finite number $p$ of previous events in the stationary time series $x_{t}$ with AR parameters $\phi_{1}, \phi_{2}, \ldots, \phi_{p}$. The Akaike criterion AIC is used to select the order $p$ of the $\operatorname{AR}(p)$ model (Salas et al., 1980).

In the next step we transform the standardized residual time series $x_{t}$ from Eq. (6) into the stochastic component $Q_{A R}$ :

$Q_{A R}(t)=x_{t}(t) \sigma_{R}=\sigma_{R}\left[\begin{array}{l}\phi_{1} \cdot x_{t}(t-1)+\phi_{2} \cdot x_{t}(t-2)+ \\ \ldots+\phi_{p} \cdot x_{t}(t-p)\end{array}\right]$.

The random time series $\varepsilon$, or the error term, is then determined by subtracting the stochastic component $Q_{A R}$ from the residual series $Q_{R}$ : 


$$
\begin{aligned}
& \varepsilon(t)=Q_{R}(t)-Q_{A R}= \\
& =Q_{R}(t)-\left[\begin{array}{l}
\phi_{1} \cdot x_{t}(t-1)+\phi_{2} \cdot x_{t}(t-2)+ \\
\ldots+\phi_{p} \cdot x_{t}(t-p)
\end{array}\right] \sigma_{R} .
\end{aligned}
$$

In order to generate a stochastic ensemble of $\varepsilon$ we use the single bootstrap model (SBM). A method of random resampling of the error term is used to generate simulations. This method is based on resampling the historical data, without modifying the values. The error term vector is presented as $\{\varepsilon\}_{t}=\left\{\varepsilon_{l}, \varepsilon_{l}, \ldots, \varepsilon_{N}\right\}$ where $t$ was a time step $(t=1, \ldots, N)$. If the SBM model is applied for single station, the stochastic ensemble of $\varepsilon$ is the following matrix

$$
\{\varepsilon\}_{t \times B}=\left\{\begin{array}{l}
\varepsilon_{1}^{1}, \varepsilon_{2}^{1}, \ldots, \varepsilon_{N-1}^{1}, \varepsilon_{N}^{1} ; \quad \varepsilon_{1}^{2}, \varepsilon_{2}^{2}, \ldots, \varepsilon_{N-1}^{2}, \varepsilon_{N}^{2} ; \\
\ldots \quad \varepsilon_{1}^{B}, \varepsilon_{2}^{B}, \ldots, \varepsilon_{N-1}^{B}, \varepsilon_{N}^{B}
\end{array}\right\} .
$$

where $B$ is the number of the bootstrap resampled vectors. In this case, the number of the resampled vectors is equal to the length of time series $Q$ (i.e. $B=N$ ). Therefore, the stochastic ensemble $\{\varepsilon\}_{t \times B}$ represents a square matrix.

The ultimate stochastic model represents the sum of the modelled components at the same time step and includes the SBM model of the resampled random vector $\varepsilon$ :

$$
\left\{Q_{m}\right\}_{t \times B}=\left\{Q_{T}\right\}_{t}+\left\{Q_{P}\right\}_{t}+\left\{Q_{A R}\right\}_{t}+\{\varepsilon\}_{t \times B} .
$$

The correlation coefficient CORR and the Nash-Sutcliffe coefficient NSE are used to evaluate the variation between the observed flows $Q$ and the modeled time series $Q_{m}$.

\section{RESULTS AND DISCUSSION}

The hydrologic stations at ten large European rivers used in this study are shown in Table 1 . We used the mean annual flow time series from these stations to analyse their stochastic structure. In the first step, presence of trend is tested by the parametric Kendal-Stuart test and the nonparametric Mann-Kendall test. The results of the trend analysis are shown in Table 2.

A statistically significant linear trend was identified at all hydrological stations at the $5 \%$ significance level $\left(|\beta|>\beta_{(\alpha=5 \%)}\right)$ except at the Orsova station. The Mann-Kendall test lead to a different conclusion: significant trend was detected at the Burghausen station only $\left(\left|Z_{M K}\right|>1.96\right)$. However, it should be noted that the trend direction and magnitude depend on the series length (Stojković et al., 2014). In further analysis the significant linear trend was removed from the flows time series $Q$.

Time series of annual flows exhibit the same characteristics as the series with long memory. The values of the Hurst coefficient $h$ at the analyzed stations prove this: Decin $(h=0.640)$, Bratislava (0.563), Cologne (0.608), Vlotho (0.741), Wasser- burg (0.541), Burghausen (0.648), Orsova (0.596), Tainionkoski (0.707), Ust Pinega (0.737), Smalininkai (0.673). The time series with long memory show in general the high frequency variations and the long-term cyclical behavior. The Fourier transform is therefore suitable for modeling hydrological time series with long memory.

The periodogram as given in Eq. (4) was used to identify the long-term periodicity in the annual flow time series. The LOESS method was applied to smooth the time series and in order to determine significant periods in the mean annual flow time series. Figure 1 shows the periodogram and the relative cumulative periodogram of the observed and the smoothed annual flows at station Orsova. The periodogram of the smoothed series in Fig. 1a has several peaks at low frequencies, which are governing the alternating long-term dry and wet periods. The level of significant periods (LSP) was set at 95\% of the cumulative periodogram (Fig. 1b).

The $L S P$ of $95 \%$ was applied to determine significant periods at all the considered hydrological stations, where the number of significant periods $q$ is used to model the periodic component by Eq. (2). The same periods were also significant according to the Fisher test at the 5\% significance level. The characteristic periods are: $7,10-12,14-15,20-25$ and 30 years. In addition, very long periods greater than 100 years were also identified at the Vlotho, Burghausen, Usti-Pinega and Smalininkai stations. The long-term periodic component is determined from Eq. (2) based on the identified significant periods. The periodic components of analysed European river show synchronous multiannual oscillations.

Annual flow residuals were modeled using the AR models of the order $p$ ranging from 5 to 7 . The order $p$ of these models was adopted according to the Akaike criterion (AIC). The stochastic component $Q_{A R}$ was consequently determined by Eq. (7). When the linear trend $Q_{T}$, periodic component $Q_{P}$ and stochastic component $Q_{A R}$ had been defined, we determined the error term (i.e. the residual series) as given by Eq. (8). The error term is a random time series with mathematical expectation $\mathrm{E}(\varepsilon)=0$ and with no significant autocorrelation at each station. In the next phase we generated a stochastic ensemble of random residuals $\{\varepsilon\}_{t \times \text { в }}$ using the single bootstrap model (Eq. 9) by performing $B=N$ simulations with resampling of the residuals at each station. The method can also be applied for the multi-site resampling of the residual series which exhibit a significant cross-correlation between each other. This approach is primarily intended for sites which are located at the same river basin and which are expected to be cross-correlated. For the sites in the same river basin the hybrid moving block bootstrap multi-site model (HMM) proposed by Srinivas and Srinivasan (2005) could be used to simulate the annual flow series. This paper considers stations over a wide European region at which the residuals do not show significant cross-correlation between the sites despite the finding that they have synchronous long-term flow fluctuations.

Table 2. Trend in annual flow series: linear regression slope coefficient $\beta$, critical value of $\beta$ at $\alpha=5 \%$ and the values of the Mann-Kendall test statistic $Z_{M K}$.

\begin{tabular}{lccclcc}
\hline Station: & $\beta$ & $\beta_{(\alpha=5 \%)}$ & $Z_{M K}$ & Station: & $\beta_{(\alpha=5 \%)}$ & $Z_{M K}$ \\
\hline Decin & 0.024 & 0.003 & 0.836 & Burghausen & -0.165 & 0.003 \\
Bratislava & 0.022 & 0.007 & 0.267 & Orsova & 0.003 & 0.003 \\
Cologne & 0.037 & 0.003 & 0.582 & Tainionkoski & -0.012 & 0.003 \\
Vlotho & -0.105 & 0.003 & -1.911 & Ust-Pinega & -0.106 & -0.087 \\
Wasserburg & -0.029 & 0.003 & -0.616 & Smalininkai & -0.053 & 0.005 \\
\hline
\end{tabular}



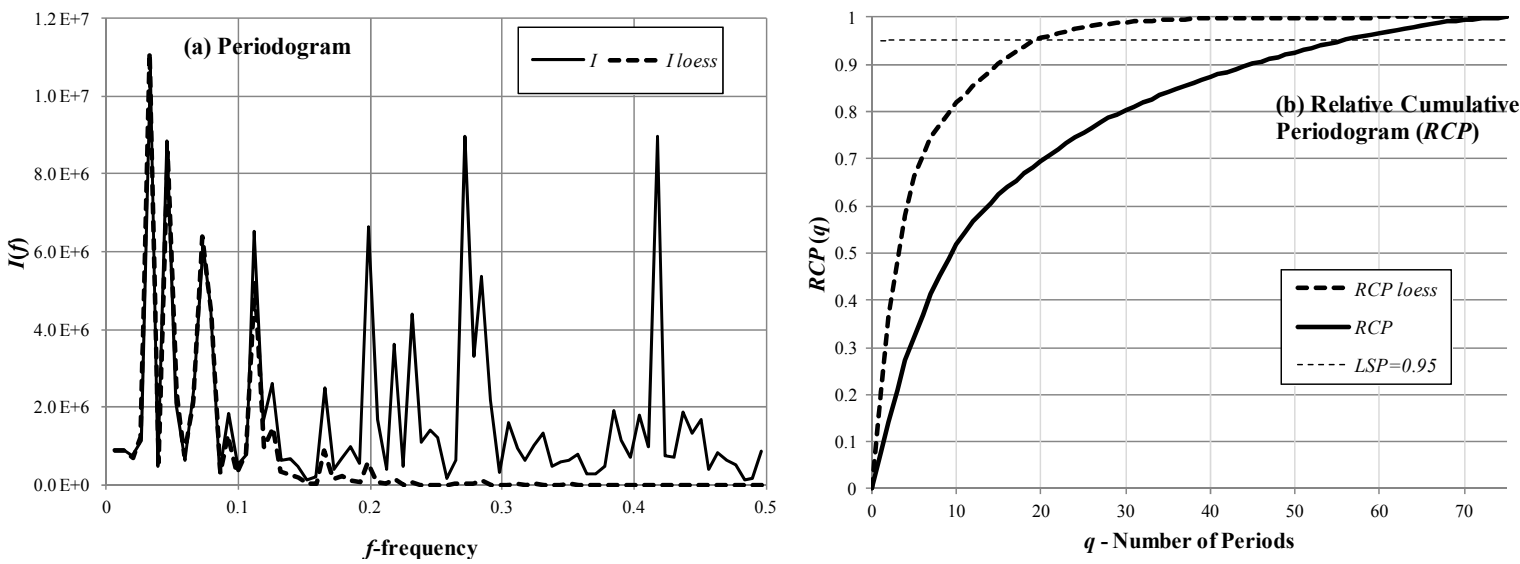

Fig. 1. Periodogram (a) and relative cumulative periodogram, RCP, (b) of the original and the smoothed annual flows at the Orsova station.

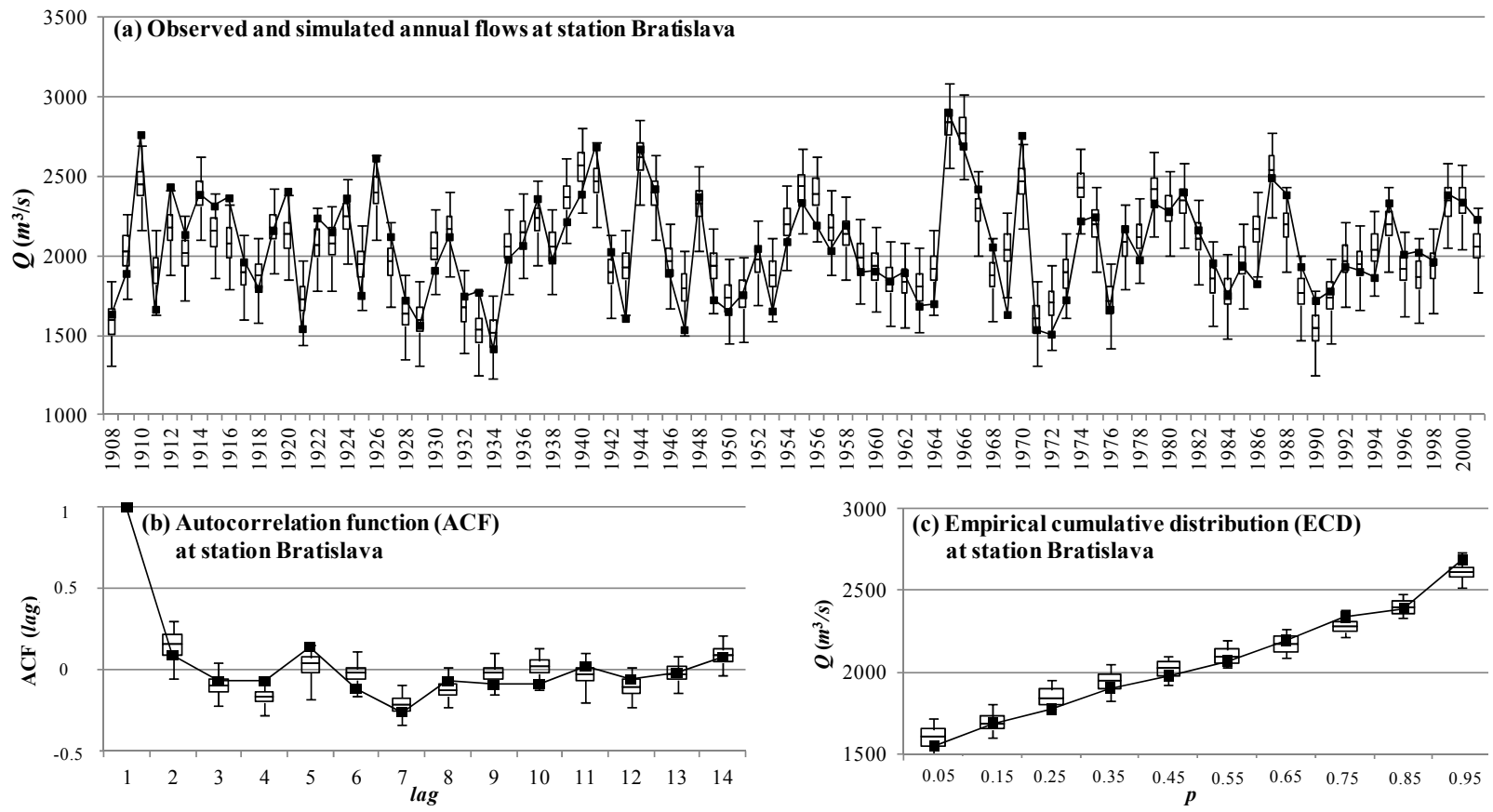

Fig. 2. Observed and simulated (a) annual flow series of the Danube at Bratislava, (b) autocorrelation function, and (c) empirical cumulative probability distribution of the annual flows. The black square markers indicate the observed series and the box plots depict the distribution of the ensemble simulations: lower, middle and upper box lines represent the first (25\%) quartile, the median (50\%) and third quartile (75\%), respectively, and the whiskers indicate the range of values in the ensemble.

The ultimate stochastic model is given by summing all the components from Eq. (10). Figure 2 presents the results of the stochastic simulation of annual flows for the Bratislava station at the Danube River. The results from the ultimate stochastic model at the other stations are shown in the Figures 4, 5 and 6.

The statistical characteristics of simulated and observed annual flows were compared, including autocorrelation function (ACF) and empirical cumulative probability (ECD). The results show that the simulated annual flows have appropriate statistical characteristics to the historical data at considered stations.

For each station the ensembles of the correlation coefficient $\{C O R R\}_{B}$ and the Nash-Sutcliffe coefficient $\{N S E\}_{B}$ were computed. It should be noted that these coefficients are generally used to evaluate goodness-of-fit between historical and simulated time series by comparing the corresponding values in time. In this study we use these coefficients as a measure of agreement between the historical series and the simulated ensemble by Eq. (10) in statistical terms. Since the explained variance of annual flows by the simulated time series without the random component ranges from $67.2 \%$ to $80.6 \%$, i.e. the random component represents a smaller part of the explained variance, the use of the CORR and NSE coefficients for the comparison between the stochastic ensemble and observed flows is justified.

Distributions of the two performance measures from the ensemble of simulated annual flows generally show small variation in the inter-quartile range (Fig. 3), although the complete range may be wider. The smallest CORR and NSE values belong to simulations that possess the greatest departure from the observed annual flow series and the greatest values correspond to those simulations which resemble the most the observed time series. The median correlation coefficient values range from just below 0.7 up to 0.8 at different stations, and the median NSE values are all above 0.5 , which could be considered satisfactory. The NSE values lower than 0.5 are obtained in a very limited number of simulations at four stations out of ten. 
Stochastic structure of annual discharges of large European rivers
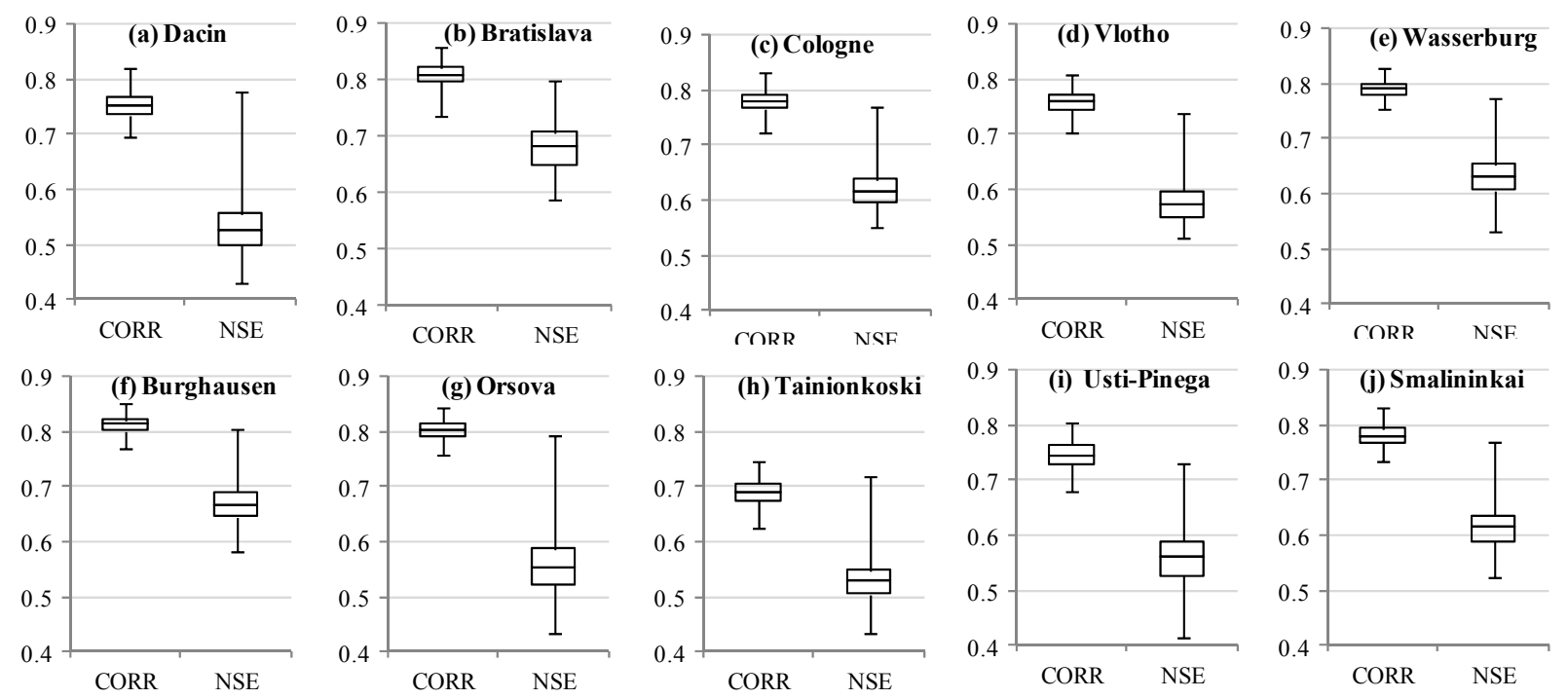

Fig. 3. Box plots of the ensembles of correlation coefficient CORR and the Nash-Sutcliffe coefficient NSE for simulated mean annual flows at the stations analysed in this paper.
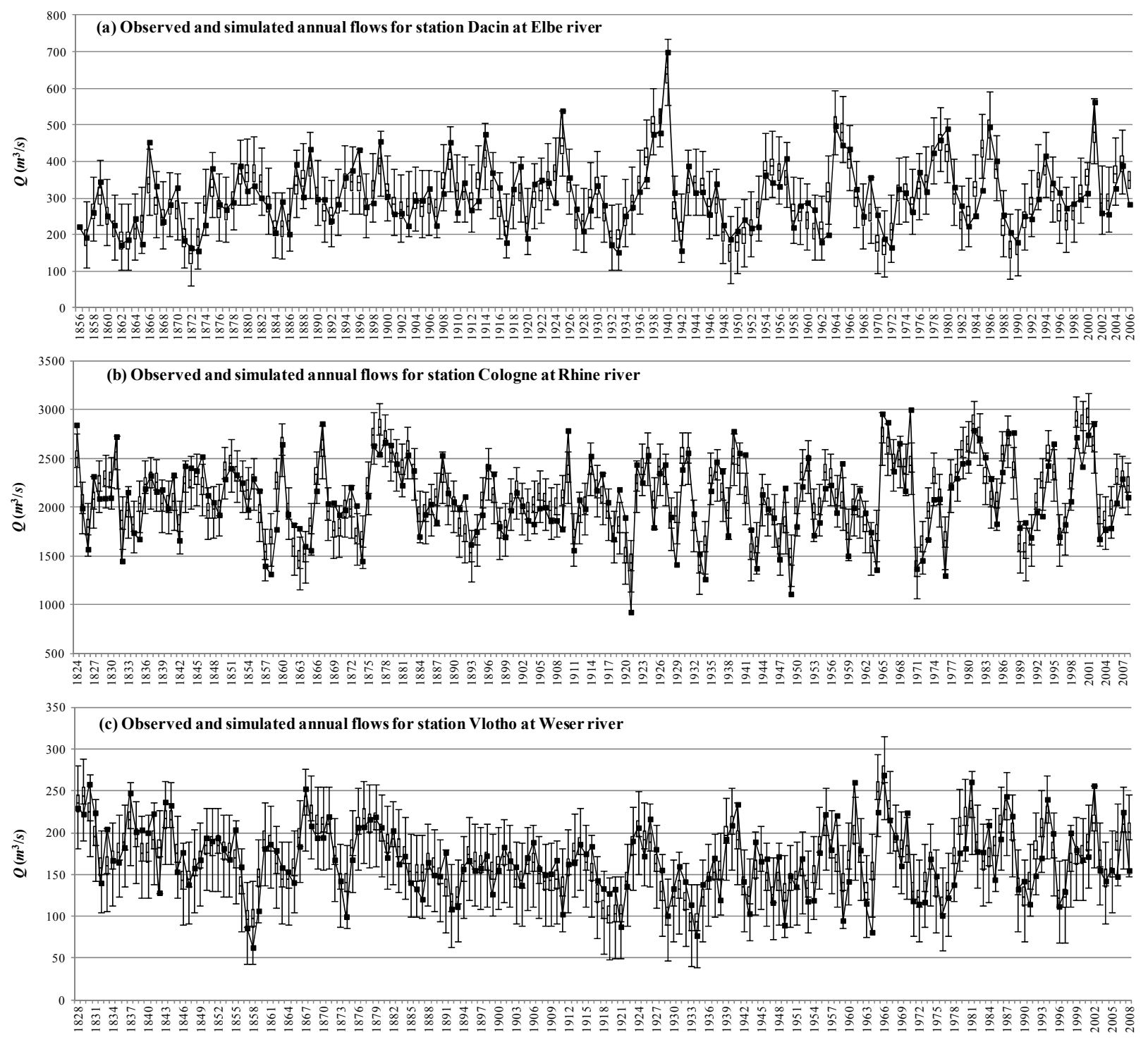

Fig. 4. Observed and simulated mean annual flows at stations: (a) Dacin, (b) Cologne, (c) Vlotho. 

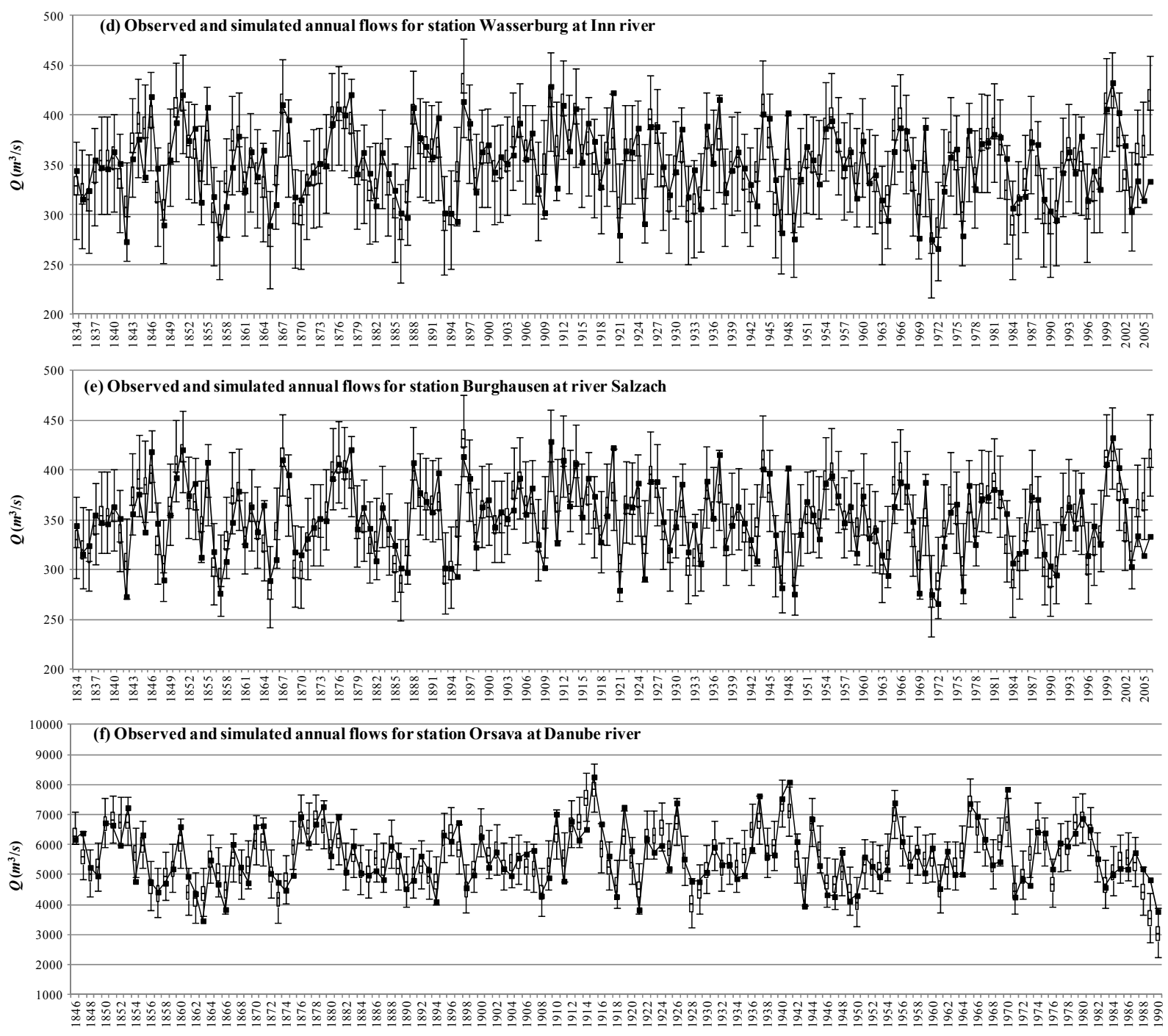

Fig. 5. Observed and simulated mean annual flows at stations: (d) Wasserburg, (e) Burghausen, (f) Orsova.

Since behavior of the simulated time series was confirmed to be similar to the observed series, they could be used for alternative hydrological scenarios in the river basin. These scenarios can be a useful tool for integrated river basin planning, especially for the basins with limited observed data.

\section{CONCLUSION}

A new stochastic model for generating hydrological series in a river basin has been introduced for simulating mean annual flows at ten large European rivers with long observation period. This model consists of two parts: the first part is inferred from the historical time series of annual flows and the second part implies generating the random time series. The random time series represents the modelling error. The first part of the stochastic model decomposes annual flows into the trend component, periodic component and stochastic component. These components are modeled by well known techniques of linear regression, the Fourier transform and linear autoregressive models, respectively. The second part is modelled by the single bootstrap model (SBM) which is used for random resampling of the error term vector. In this way a stochastic ensemble of error terms is generated at a single hydrological station. The ultimate stochastic model aggregates the first and the second part at the same time step. This produces an ensemble of stochastic simu- lations of annual flows at considered stations. The simulated annual flows have appropriate statistical characteristics of the observed flows. The autocorrelation function and empirical cumulative probability distribution are used for model verification. Also, the correlation coefficient and the Nash-Sutcliffe coefficient are used to explore variation in the similarity of the simulated ensemble to the historical data. The conclusion is that stochastic simulated flows can be used for hydrological simulations in a river basin.

Water resources planning and management can be in general seen as stochastic problems. Integrated river basin planning and water management studies involve stochastic simulation of annual flows in a basin. In most cases hydrological observations are limited in length. It is necessary to develop stochastic simulation models for solving issues such as reservoir sizing and defining operational rules for managing complex water infrastructure. The purpose of the present stochastic model is to contribute to these issues. In addition, this stochastic model can be used to generate different hydrological scenarios in the river basin. The trend and periodic components are functions of time which could be extrapolated for the future time period in the $21^{\text {st }}$ century. This is an alternative approach in comparison to the hydrological projections based on the climate parameters under greenhouse gases emission scenarios. 

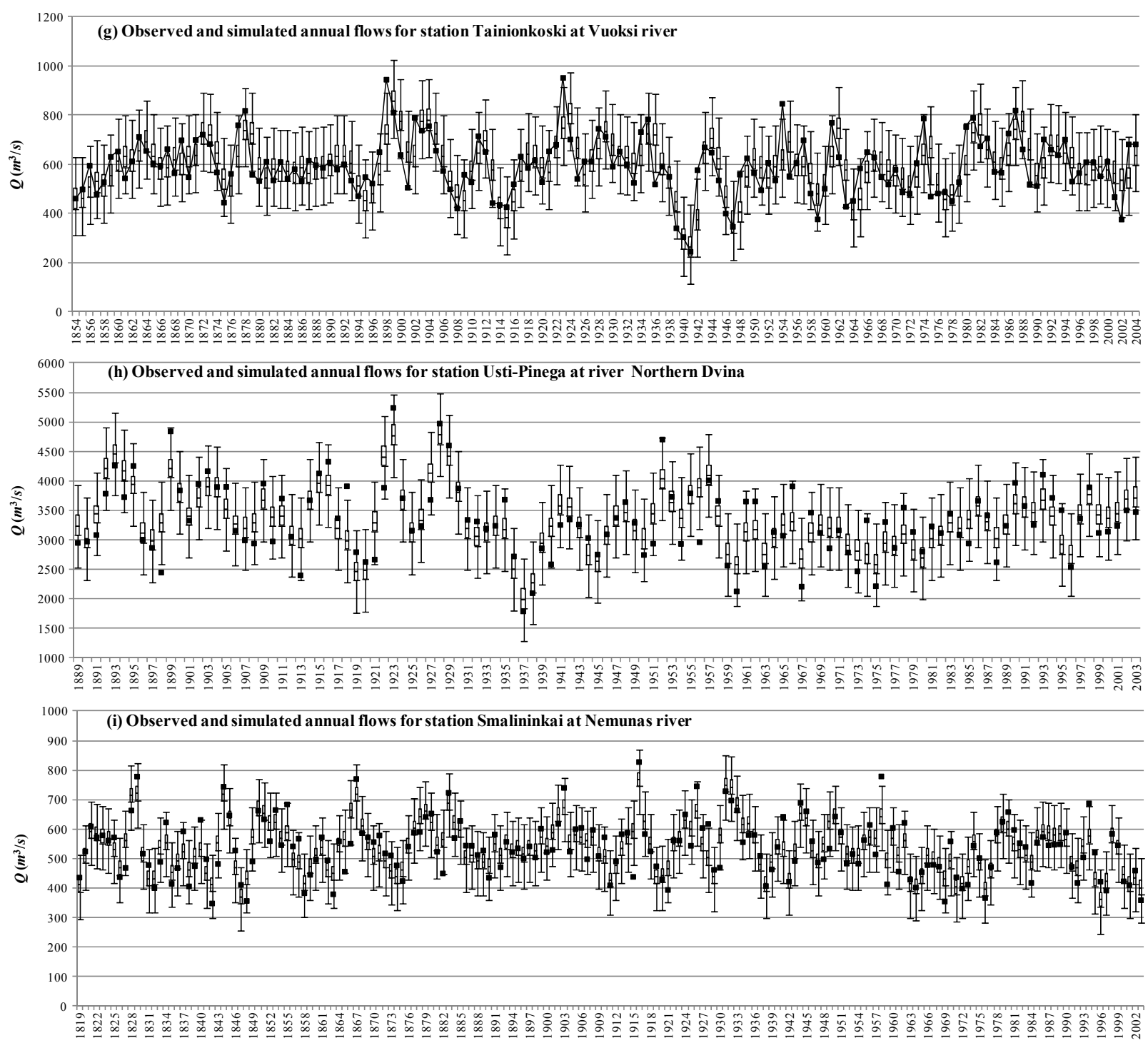

Fig. 6. Observed and simulated mean annual flows at stations: (g) Tainionkoski, (h) Ust-Pinega, (i) Smalininkai.

Acknowledgments. The research presented in this paper is funded by Serbia's Ministry of Education and Science as part of an ongoing scientific project titled "Assessment of Climate Change Impact on Water Resources in Serbia" (TR-37005, 2011-2014). The authors are also grateful to two anonymous reviewers for their constructive comments and suggestions for improving this paper.

\section{REFERENCES}

Birsan, M.V., Molnar, P., Burlando, P., Pfaundler, M., 2005. Streamflow trends in Switzerland. Journal of Hydrology, $314,1-4,312-329$.

Blasco, N., Santamaria, R., 1996. Testing memory patterns in the Spanish stock market. Applied Financial Economics, 6, 5, 401-411.

Box, G.E.P., Jenkins, G., 1970. Time Series Analysis, Forecasting and Control. First Ed. Holden day Inc., San Francisco.

Douglas, E.M., Vogel, R.M., Kroll, C.N., 2000. Trends in floods and low flows in the United States: impact of spatial correlation. Journal of Hydrology, 240, 1-2, 90-105.
Fendekova, M., Pekarova, P., Fendek, M., Pekár, J., Škoda, P., 2014. Global drivers effect in multi-annual variability of runoff. J. Hydrol. Hydromech., 62, 3, 169-176.

Hanggi, P., Weingartner. R., 2011. Inter-annual variability of runoff and climate within the Upper Rhine River Basin, 1808-2007. Hydrological Sciences Journal, 56, 1, 34-50.

Hannaford, J., Buys, G., Stahl, K., Tallaksen, L.M., 2013. The influence of decadal-scale variability on trends in long European streamflow records. Hydrol. Earth Syst. Sci., 17, 2717-2733.

Hurst, H., 1951. Long term storage capacity of reservoirs, Transactions of the American Society of Civil Engineers. 6, 770-799.

Kendall, M.G., Stuart, A., 1966. Anvanced of Statistics. vol 3. Design and Analysis and Time Series. Hafner Publ. Co., New York.

Khaliq, M.N., Ouarda, T., Gachon, P., Sushama, L., StHilaire, A., 2009. Identification of hydrological trends in the presence of serial and crosscorrelations: A review of selected methods and their application to annual flow regimes of Canadian rivers. Journal of Hydrology, 368, 1$4,117-130$ 
Kundzewicz, Z.W., Bates, B., Wu, S., Palutikof, J. 2008. Climate change and water, Intergovernmental Panel on Climate Change. IPCC Technical Paper VI. IPCC Secretariat, Geneva, $210 \mathrm{p}$.

Labat, D., 2006. Oscillations in land surface hydrological cycle. Earth and Planetary Science Letters, 242, 1-2, 143 154.

Labat, D., Godderis, Y., Probst, J.L., Guyot, J.L., 2004. Evidence for global runoff increase related to climate warming. Advances in Water Resources, 27, 6, 631-642.

Machiwal, D., Jha, M.K., 2006. Time series analysis of hydrologic data for water resources planning and management: a review. J. Hydrol. Hydromech., 54, 3, 237-257.

Milly, P.C.D., Dunne, K.A., Vecchia, A.V. 2005. Global pattern of trends in streamflow and water availability in a changing climate. Nature, 438, 7066, 347-350.

Mudelsee, M., 2010. Climate Time Series Analysis: Classical Statistical and Bootstrap Methods. Springer, Dordrecht, $474 \mathrm{p}$.

Pekarova, P., Pekar, J., 2006. Long-term discharge prediction for the Turnu Severin station (the Danube) using a linear autoregressive model. Hydrological Processes, 20, 5, 1217-1228.

Pekarova, P., Miklanek, P., Pekar, J., 2003. Spatial and temporal runoff oscillation analysis of the main rivers of the world during the 19th-20th centuries. Journal of Hydrology, 274, 1-4, 62-79.

Pekarova, P., Miklanek, P., Pekar, J., 2006. Long-term trends and runoff fluctuations of European rivers. Climate variability and change - hydrological impacts. In: Proc. 5th FRIEND World Conference, Havana, Cuba.
Probst, J., Tardy, Y., 1987. Long range streamflow and world continental runoff fluctuation since the beginning of this century. Journal of Hydrology, 94, 3-4, 289-311.

Salas, J.D., Delleur, J.W., Yevjevich, V., Lane, W.L., 1980. Applied Modeling of Hydrologic Time Series. Water Resources Publications, Littleton, Colorado, USA, 484 p. (2nd Printing 1985, 3rd Printing, 1988)

Shuster, A., 1887. On lunar and solar periodicities of earthquakes. Proc. Roy. Soc., pp. 455-465.

Srinivas, V.V., Srinivasan, K., 2005. Hybrid moving block bootstrap for stochastic simulation of multi-site multiseason streamflows. Journal of Hydrology, 302, 1-4, 307330 .

Stahl, K., Hisdal, H., Hannaford, J., Tallaksen, L.M., Van Lanen, H.A.J., Sauquet, E., Demuth, S., Fendekova, M., Jodar, J., 2010. Streamflow trends in Europe: evidence from a dataset of near-natural catchments. Hydrology and Earth System Sciences. 14, 12, 2367-2382.

Stojković, M., Ilić, A., Prohaska, S., Plavšić, J., 2014. Multitemporal analysis of mean annual and seasonal stream flow trends, including periodicity and multiple non-linear regression. Water Resources Management, 28, 12, 43194335 .

Stojković, M., Prohaska, S., A. Koprivica., 2012. Analysis of Trends and Cycles in Longest Hydrometeorological Time Series in the World. BALWOIS, Ohrid, Macedonia.

Yevjevich, V. 1972. Stochastic processes in hydrology. Water Resources Publications, Fort Collins, Colorado, USA.

Received 5 June 2014 Accepted 13 November 2014 